PREFACE

Series B

\title{
Special Issue: Integer Programming and Combinatorial Optimization (IPCO) 2020
}

\author{
Daniel Bienstock ${ }^{1} \cdot$ Giacomo Zambelli $^{2}$ \\ Published online: 10 February 2022 \\ ๑ Springer-Verlag GmbH Germany, part of Springer Nature and Mathematical Optimization Society 2022
}

This volume of MPB (Mathematical Programming, Series B) contains 25 high-quality articles in the area of integer programming and combinatorial optimization. Extended abstracts of these articles have previously appeared in the proceedings of the 20th Conference on Integer Programming and Combinatorial Optimization (IPCO), held June 8-10, 2020, in London, UK. The proceedings volume, also published by Springer in the Lecture Notes in Computer Science series (volume 12,125), comprises 33 extended abstracts (out of 126 submissions) that were selected and presented at IPCO 2020.

The IPCO conference is under the auspices of the Mathematical Optimization Society, and is held every year. Until 2018, years divisible by three (in which the International Symposium on Mathematical Programming took place) were skipped. The conference is a forum for researchers and practitioners working on various aspects of integer programming and combinatorial optimization. The aim is to present recent developments in theory, computation, and applications in these areas. The first IPCO conference took place at the University of Waterloo in May 1990. More information on IPCO and its history can be found at www.mathopt.org/?nav=ipco.

All authors of extended abstracts that were accepted for IPCO 2020 were invited to submit full journal papers to be considered for publication in this special volume of MPB. As compared to the IPCO extended abstracts, the full journal papers are longer, containing full proofs, extensions of the preliminary results, and additional details. The submitted papers underwent a rigorous refereeing process; this volume contains the papers that were accepted following appropriate revisions. These papers that now appear represent a snapshot of the very best of an exciting and vibrant domain within mathematical optimization.

\footnotetext{
$凶$ Daniel Bienstock

dano@columbia.edu

Giacomo Zambelli

G.Zambelli@1se.ac.uk

1 Department of Applied Physics and Mathematics, Columbia University, New York, USA

2 Department of Mathematics, London School of Economics and Political Science, London, UK
} 
We thank all authors and in particular the referees who reviewed the papers thoroughly. We also would like to thank Sven Leyffer, the Editor-in-Chief of MPB, for his excellent cooperation.

Publisher's Note Springer Nature remains neutral with regard to jurisdictional claims in published maps and institutional affiliations. 